The UK economy looks to be at a turning point with unemployment on the rise. Growth is faltering as the government thrust to demand is weakening and all the talk is of the housing market declines, continued feeble business investment and the worst consumer sales figures for 20 years. There is no sign whatever that a vibrant, deregulated private sector is about to take up the slack. The days of Tony Blair and Chancellor Gordon Brown preaching smugly to their European counterparts about the virtues of flexibility may be numbered.

\title{
Reference
}

Baker, Dean/Glyn, Andrew/Howell, David/Schmitt, John (2005): Labour Market Institutions and Unemployment: A Critical Assessment of the Cross-country Evidence, in: Howell, David (ed.), Fighting Unemployment. The Limits of Free Market Orthodoxy, Oxford University Press: New York, pp. 72-II8

\section{Noch eine Reform: Der neue Tarifvertrag im öffentlichen Dienst Consuela Ramos*}

Ohne Zweifel: Die Bundesrepublik Deutschland befindet sich im »Reformfieber«. Die Diskussion um angeblich notwendige Veränderungen der ökonomischen und sozialen Institutionen Deutschlands ist zu einem Macht generierenden Diskurs geworden. Politische Akteur|inn|e|n müssen sich als »reformfähig « darstellen, um überhaupt noch in der Öffentlichkeit wahrgenommen zu werden oder gar Einfluss auf die konkrete Ausgestaltung von Veränderungen nehmen zu können.

Im Februar dieses Jahres bewiesen die Tarifpartner|innen des öffentlichen Dienstes, an ihrer Spitze Innenminister Otto Schily und der Vorsitzende der Vereinten Dienstleistungs-

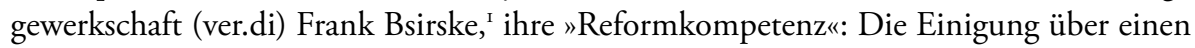
neuen Tarifvertrag für die Beschäftigten des Bundes und der Kommunen - die Länder wehren sich derzeit noch gegen die Übernahme des Ergebnisses - wurde von Bsirske als "Jahrhundert-Reform " ${ }^{2}$ gefeiert. Die meisten Kommentator|inn|en waren nicht ganz so

* Göttingen.

I Wichtige weitere Tarifparteien in den Verhandlungen um den Tarifvertrag für den öffentlichen Dienst (TVöD) waren auf Seite der Beschäftigten die dbb tarifunion, auf Seite der Verwaltungen die kommunalen Arbeitgeberverbände. Die dbb tarifunion führt die Tarifverhandlungen für Fachgewerkschaften und Berufsverbände des öffentlichen Dienstes und seiner privatisierten Bereiche.

2 Frankfurter Allgemeine Zeitung vom I0.02.2005. 
euphorisch, goutierten allerdings den Abschluss zumeist als Beitrag zu einer grundsätzlichen Reform der öffentlichen Verwaltung in Deutschland. ${ }^{3}$

\section{Prospektierte Elemente der Tarifreform}

Die Einigung, die unter der Bezeichnung »Sondierungsergebnis« publiziert wurde, betraf zunächst nur einige der von den Tarifparteien als zentral angesehenen Punkte:

I. Die Vereinfachung des Tarifwerks

Die alte Bezahlungssystematik des Bundesangestelltentarifs (BAT) wird bereits zum OI.IO.2005 durch eine neue Entgelt-Tabelle mit I5 Gruppen und sechs Entwicklungsstufen ersetzt. Die Unterscheidung zwischen Arbeiter|inne|n und Angestellten entfällt.

2. Leistungsbezug in der Grundvergütung

Die Bewegung in den sechs Entwicklungsstufen erfolgt auf Basis der Dauer der Zugehörigkeit zum öffentlichen Dienst, ist jedoch von der Leistung der|des Beschäftigten abhängig. Bei überdurchschnittlichen Leistungen kann eine höhere Stufe früher gewährt werden, bei unterdurchschnittlichen Leistungen kann die Einstufung in die nächste Entwicklungsstufe ausgesetzt werden.

3. Aus Zulagen und Zuschlägen finanzierte Leistungskomponente

Allgemeine Zulagen sowie Orts- und Sozialzuschläge entfallen (mit wenigen Ausnahmen). Aus diesem Budget wird eine Leistungskomponente finanziert, die als Zulage oder Prämie bezahlt wird und im Jahr 2007 ein Prozent der Grundvergütung betragen soll. Die Leistungskomponente soll auf acht Prozent ansteigen, wobei hierfür kein zeitliches Ziel genannt ist. Bis 2007 werden aus dem frei werdenden Budget außerdem Einmal- und Sonderzahlungen sowie eine Anhebung des Grundentgelts für die im Tarifgebiet Ost Beschäftigten finanziert.

\section{Neuregelung der Arbeitszeit}

Die tarifliche Arbeitszeit beträgt für alle Bundesbeschäftigten 39 Stunden. Im Bereich der kommunalen Arbeitgeber|innen sind betriebliche Ausweitungen auf 40 Stunden möglich.

Der neue Tarifvertrag für den öffentlichen Dienst (TVöD) wird auf Basis dieses "Sondierungsergebnisses " in verschiedenen Projektgruppen ausgearbeitet und soll dann bis mindestens Ende 2007 gelten. ${ }^{4}$

3 Erste Überlegungen zu einer stärkeren Leistungsorientierung bei der Vergütung im öffentlichen Sektor gab es in der BRD bereits 1973 (vgl. zur Übersicht Tondorf 1997: 7 ff.). Diese Überlegungen scheiterten immer wieder - zuletzt 1997 durch die Nicht-Umsetzung bundestariflich vereinbarter Möglichkeiten auf Landesebene - am Widerstand der Gewerkschaften.

4 Der vorliegende Artikel entstand im Mai 2005. Der TVöD wurde mittlerweile am 13.09.2005 von den Tarifvertragsparteien mit den im Sondierungsergebnis festgelegten Eckpunkten unterzeichnet. In dem seit Mai 2005 vorliegenden Entwurf für ein »Gesetz zur Reform der Struktur des öffentlichen 
Die Reaktionen auf die erzielten Verhandlungsergebnisse waren in der Öffentlichkeit, beispielsweise in den renommierten Tageszeitungen, durchweg verhalten positiv, die beteiligten Akteur|inn|e|n wurden hier und da sogar ausdrücklich für ihren »Reformwillen" gelobt.

Kritik wurde einerseits von den Verhandlungsführer|inne|n der Bundesländer formuliert, die eine weitere Verlängerung der Arbeitszeit bzw. entsprechende Öffnungsklauseln forderten. Unklar ist noch, wie dieser Konflikt gelöst werden wird, zumal ver.di sich verpflichtet hat, einen hinsichtlich der Arbeitszeit für die Länder günstigeren Abschluss auf Bund und Kommunen zu übertragen. ${ }^{5}$

Linke Gewerkschafter|innen und Autor|inn|en stellten dagegen die "substanziellen Verschlechterungen« für die Beschäftigten heraus: ${ }^{6}$ faktische Reallohneinbußen, Arbeitszeitverlängerung im Westen, Öffnungsklauseln etc. Die Gruppierung »Bundesweite ver.diLinke« spricht von einem »katastrophalen Tarifabschluss « und beklagt gemeinsam mit anderen ver.di-Untergruppen auch ein Defizit innergewerkschaftlicher Demokratie im Prozess der Aushandlung des TVöD. ${ }^{7}$

Diese in der kritischen Diskussion herausgearbeiteten Punkte betreffen allerdings nur die Rahmendaten der Tarifeinigung. Die hinter dem Begriff des "Reformprojekts« stehenden Veränderungsimpulse lassen sich kaum von einer verlängerten Arbeitszeit oder der Einführung einer neuen Entgelttabelle erwarten. Den Reformimpetus sehen die Tarifparteien offensichtlich in der neu eingeführten Leistungsorientierung, die dafür sorgen soll, dass die Verwaltung sich weiter modernisiert und ihre Arbeit stärker an den Anforderungen der Bürger|innen ausrichtet - wie es in der Präambel der »Sondierungsergebnisse« heißt.

Auch wenn für die neu eingeführte Leistungsorientierung nur Eckpunkte vereinbart wurden und die konkrete Ausgestaltung auf betrieblicher Ebene stattfinden soll, so lassen sich doch bereits heute wesentliche Implikationen der Neuregelung erkennen.

\section{Leistungsbezogene Vergütung: Erfahrungen aus privatwirtschaftlichen Unternehmen}

Leistungsbezogene Prämien oder Zulagen sind seit langem ein personalwirtschaftliches Instrument in privaten Unternehmen. ${ }^{8}$ Insbesondere Unternehmen des primären und sekundären Sektors können auf langjährige Erfahrungen mit Akkord-Entlohnung oder leistungsbezogenen Zulagensystemen verweisen. In Dienstleistungsunternehmen waren dagegen leistungsorientierte Vergütungselemente, bis weit in die I99oer Jahre hinein, auf Führungskräfte

Dienstrechts«, das u.a. die zukünftige Bezahlung der Beamt|inn|en regeln wird, ist die Einführung eines Leistungsbezugs entsprechend der Regelungen des TVöD vorgesehen. Die folgende Argumentation lässt sich somit auch auf den Bereich des Dienstrechts übertragen.

5 Diese "Meistbegünstigungsklausel « ist Teil der »Sondierungsergebnisse«.

6 Die umfangreichste Dokumentation entsprechender Beiträge findet sich auf den Internetseiten von labournet: http://www.labournet.de/diskussion/gewerkschaft/tarifo4/verdi/oedo4.html.

7 Vgl. »Erklärung der bundesweiten ver.di-Linken« zum Abschluss ihrer Konferenz vom I6. bis 17.04.2005, dokumentiert auf http://www.labournet.de/diskussion/verdi/bundeslinkeo5.html.

8 Als Überblickswerk vgl. Zander/Femppel (2000). 
und auf Mitarbeiter|innen aus Vertriebsbereichen beschränkt. ${ }^{9}$ Prototyp der tarifvertraglichen Einführung leistungsbezogener Entgelte für alle Beschäftigten eines Dienstleistungsunternehmens war die 1998 zwischen dem IT-Systemhaus debis und der IG Metall geschlossene Vereinbarung. ${ }^{\text {Io }}$ Diesem Tarifvertrag folgten inhaltlich ähnliche Regelungen unter anderem im Telekom-Konzern, im Konzern der Deutschen Post sowie bei Tochterunternehmen der Deutschen Bahn AG. ${ }^{\text {II }}$ Dabei wurde immer öfter von der nun auch im TVöD genutzten Möglichkeit Gebrauch gemacht, lediglich die Rahmenbedingungen, insbesondere den prozentualen Anteil der leistungsbezogenen Entgelte und deren Finanzierung, tarifvertraglich festzulegen und Detaillösungen dezentral zu vereinbaren.

Unternehmen verfolgen mit solchen Lösungen in der Regel zwei Ziele: Erstens versuchen sie durch leistungsorientierte Bezahlungssysteme zusätzliche Anreize zur Leistungserbringung zu setzen. Zweitens versuchen Unternehmen ihre fixen Personalkosten zu variabilisieren und mit dem Unternehmenserfolg zu synchronisieren. Während in den r99oer Jahren in deutschen Unternehmen mehrheitlich Systeme installiert wurden, die eine Prämie "on top« (also zusätzlich zum Grundgehalt) ermöglichten, sind seit 2002 mehr und mehr Fälle zu beobachten, in denen Budgets z.B. für Überstunden, Wochenendarbeit, Sonderzahlungen etc. zu Budgets für Leistungsprämien umgewidmet werden. Im Gegenzug vereinbaren die Sozialpartner in solchen Fällen oft Ausschüttungsgarantien. Mithin wird die gesamte Entgeltsumme nicht kleiner, sondern nur anders - nämlich leistungsdifferenziert verteilt (vgl. Göckel et al. 2003). Auch der TVöD ist nach diesem Prinzip konstruiert. In vielen Fällen entfällt daher heute das zweite Motiv der Personalkostenflexibilisierung. Ein Nutzen leistungsorientierter Entgeltsysteme kann dann nur durch den zusätzlichen Leistungsanreiz und dadurch erzielbare Produktivitätssteigerungen, ${ }^{\mathrm{I2}}$ nicht aber durch unmittelbare Einsparungen bei den Lohnkosten begründet werden.

Die Diskussion über die motivationale Wirkung leistungsorientierter Vergütung hat unter deutschen Manager|inne|n und Personalverantwortlichen durch pointierte Beiträge in den letzten Jahren wieder an Beliebtheit gewonnen. Während die Gegner|innen leistungs-

9 Eine Feinanalyse könnte noch einmal zwischen leistungs- und erfolgsorientierten Entgelten unterscheiden. Für die im Folgenden zu entwickelnde Argumentation wird die Zusammenfassung unter dem Begriff der Leistungsorientierung ausreichen.

Io Als Überblick über den Tarifvertrag vgl. Schulten (1998).

II Der Trend beschränkt sich nicht auf die genannten, ehemals staatlichen Unternehmen. Kurdelbusch (2002) untersucht 90 Großunternehmen in Deutschland und kommt zu dem Ergebnis, dass 70 Prozent dieser Unternehmen mit leistungsorientierten Vergütungskomponenten arbeiten. Kaar/ Grünell (200I) geben für I999/2000 die Zahl von 46 Prozent deutscher Unternehmen mit leistungsorientierter Bezahlung für alle Mitarbeiter|innen in ihrer Stichprobe an.

I2 Im Sinne akteurstheoretischer Ansätze kann man leistungsorientierte Vergütungssysteme als ex-post-Verhandlung (bzw. »durchgehende Verhandlung « im Sinne von Williamson (I990: 33)) eines ex ante nicht hinreichend spezifizierten Arbeitsvertrages und einer Leistungserbringung unter asymmetrischer Information verstehen. Leistungsorientierte Vergütungssysteme senken hiernach die Transaktionskosten und erhöhen die Kongruenz zwischen den Interessen von principal (dem Unternehmen) und agent (der|dem Arbeitnehmer|in). Dies ist auch die theoretische Grundlage so genannter effizienzlohntheoretischer Ansätze; vgl. zur Übersicht die Beiträge in Akerlof/Yellen (Hg.) (1986). 
bezogener Vergütung diese als Ausdruck einer »Misstrauenskultur« interpretieren, welche jegliche Leistungsbereitschaft lähme (Sprenger 2005: $45 \mathrm{f}$.), verweisen die Befürworter|innen auf die - aus ihrer Perspektive - offensichtliche Bereitschaft einer Mehrzahl der Beschäftigten, für bessere Bezahlung auch mehr zu leisten. ${ }^{13}$ Die dichotomisierende Diskussion ignoriert allerdings die betriebliche Realität. Die Einführung moderner leistungsorientierter Vergütungssysteme, die in ihrer Systematik dem TVöD ähnlich sind, impliziert weit reichende Veränderungsprozesse: Veränderungen der innerorganisatorischen Kommunikation wie auch des Führungsverhaltens sind als kritische Erfolgsfaktoren für die Einführung erkannt (vgl. Göckel et al. 2003). ${ }^{\text {I4 }}$

Die Detailfrage nach den motivationalen Wirkungen leistungsorientierter Vergütung wäre zu vernachlässigen, wenn sich empirisch zeigen ließe, dass leistungsbezogene Entgeltsysteme die Unternehmens-Performance verbessern. Untersuchungen, die der Komplexität dieser Frage gerecht würden, liegen allerdings nicht vor. Beim momentanen Stand der empirischen Forschung bleibt zudem die Kausalität unklar: Machen leistungsorientierte Vergütungssysteme Unternehmen erfolgreich oder implementieren erfolgreiche Unternehmen solche Vergütungssysteme ${ }^{\text {I5 }}$ Der »Boom « leistungsorientierter Vergütung gründet sich daher bislang vor allem auf positive Fallbeispiele und die Plausibilität für das Alltagsbewusstsein. ${ }^{16}$ Dabei wird - durchaus plausiblerweise - unterstellt, dass eine engere Verbindung von strategischen Zielen des Unternehmens, den daraus abgeleiteten Zielen für Führungskräfte und Mitarbeiter|innen auf der einen Seite und den leistungsorientierten Vergütungselementen der Führungskräfte und Mitarbeiter|innen auf der anderen Seite eine größere Kohärenz zwischen Unternehmenszielen und tatsächlichem Handeln der Mitarbeiter|innen herstellt. Der Erfolg eines leistungsorientierten Vergütungssystems hängt in diesem Sinne davon $\mathrm{ab}$, inwiefern es gelingt, von den Mitarbeiter|inne|n genau jene Leistungen zu bekommen, die den Unternehmenserfolg maximieren. Entscheidend wird, wie »Leistung « überhaupt definiert und objektiviert gemessen werden kann.

I3 Im Sinne von Coleman (I995: 90 f.) verweisen die Befürworter|innen einer konjunkten - sozusagen "freiwilligen « - Herrschaft auf Basis von Vertrauen und Einsicht auf deren Überlegenheit gegenüber einer disjunkten Herrschaft - nämlich der gegen Bezahlung. Hinsichtlich der Transaktionskosten für die Überwachung von Handlungen ist das offensichtlich völlig korrekt. Die Frage ist jedoch, ob eine konjunkte Herrschaft in modernen Unternehmen effizient herzustellen ist, d.h. ob nicht die Kosten zum Aufbau von konjunkten Herrschaftsbeziehungen größer als die Nachteile durch disjunkte Herrschaftsformen sind. Die Exponent|inn|en der praxisbezogenen Diskussion, die Manager|innen adressiert (vgl. z.B. Dressler 2000 und Sprenger 2005), haben diese Frage bislang noch nicht thematisiert. I4 Es geht also um eine Optimierung des Verhältnisses von - wiederum im Sinne von Coleman (I995: 90 f.) - konjunkten und disjunkten Elementen der Herrschaft zur Senkung der Transaktionskosten im Prozess der Leistungserbringung.

I5 Vgl. hierzu Kurdelbusch (2002: 83 ff.).

I6 Zwei weitere mögliche Gründe für diesen Trend lassen sich in Analogie an Neubergers (1989) Kritik der Assessment-Center-Methodik entwickeln. Wie bei den Assessment Centern handelt es sich auch bei leistungsorientierten Vergütungssystemen um ein Instrument, das Macht - nämlich der Führungskräfte über die Mitarbeiter|innen - stabilisiert und mit dem Unternehmen ihre Modernität und Leistungsorientierung zeigen können. 


\section{Definition und Messung von Leistung: Neue Anforderungen an die Führungskompetenz}

In der Physik wird Leistung als Energie pro Zeit definiert. Leider macht die Messung des Kalorienverbrauchs von Beschäftigten betriebswirtschaftlich wenig Sinn. Unternehmen müssen also andere Leistungskriterien finden. Die bereits erwähnte Verbreitung leistungsorientierter Entgelt-Bestandteile bei Führungskräften, im Vertrieb sowie im produzierenden Gewerbe ist eine Konsequenz der relativ einfachen Messbarkeit von Leistung in diesen Bereichen, beispielsweise in Form des Umsatzes, des betriebswirtschaftlichen Ergebnisses oder bestimmter Stückzahlen. Solche einfachen Messkriterien fehlen für Mitarbeiter|innen in den Support-Bereichen von privaten Unternehmen ebenso wie für die Beschäftigten in öffentlichen Unternehmen und Organisationen.

Für die Praxis leistungsorientierter Vergütung stehen zwei Mechanismen der Leistungsmessung zur Verfügung:

\section{Zielvereinbarungen}

Zielvereinbarungen werden zwischen Führungskräften und Mitarbeiter|inne|n - sozusagen als dezentrale Ergänzung des Arbeitvertrags - geschlossen. Sie definieren ex ante die in einem bestimmten Zeitraum, meist einem Jahr, zu erreichenden Ziele. Am Ende dieses Zeitraums sollen idealerweise Führungskraft und Mitarbeiter|innen die Zielerreichung gemeinsam feststellen. Der Zielerreichungsgrad dient dann als Messgröße für die im betrachteten Zeitraum erbrachte Leistung.

2. Leistungsbeurteilungen/-bewertungen ${ }^{17}$

Leistungsbeurteilungen sollen in gemeinsamen Gesprächen zwischen Führungskräften und Mitarbeiter|inne|n stattfinden und die in einer Periode erbrachte Leistung ex post anhand festgelegter Beurteilungskriterien messen. Auch bei diesem Verfahren findet die Leistungsmessung idealerweise im Konsens zwischen Führungskraft und Mitarbeiter|in statt.

Die moderne Praxis leistungsorientierter Bezahlung basiert auf einem kooperativen, auf Konsens mit den Beschäftigten ausgelegten Führungsmodell. ${ }^{18}$ Das mag auf den ersten Blick idealistisch wirken, ist es aber nicht: Die Existenz widersprüchlicher Interessen zwischen Beschäftigten und dem Unternehmen wird ja nicht negiert, sondern die Bearbeitung der aus diesen resultierenden Konflikte auf die unterste Management-Ebene verlagert. Führungskräfte sind gezwungen, sich mit Mitarbeiter|inne|n über erwartete und erbrachte Leistungen auseinanderzusetzen, und das vor allem mit zwei Konsequenzen: Erstens müssen dezentral objektivierte Leistungskriterien gefunden werden, die einerseits dem Unternehmenserfolg dienen und die andererseits von den Mitarbeiter|inne|n als Kriterien für leistungsorientierte Vergütungskomponenten akzeptiert werden. Zweitens werden Führungskräfte in einen Leis-

I7 Im Zusammenhang mit dem TVöD wird der Begriff der "Systematischen Leistungsbewertung « verwendet, vermutlich zur Abgrenzung gegenüber den bisher im Rahmen des BAT und des Beamtenrechts existierenden Beurteilungssystemen.

I8 Als Beleg für die Popularität dieses Management-Trends vgl. auch Sprenger (2005). 
tungsdiskurs mit ihren Mitarbeiter|inne|n gezwungen: Die resultierende Leistungsdifferenzierung soll gewissermaßen diskursiv zur Motivation der Beschäftigten genutzt werden: Mehr Kommunikation über Ziele und Verbesserungschancen, mehr Feedback für Beschäftigte und Führungskräfte, mehr gemeinsame Anstrengungen zur Steigerung der Leistung sollen im Idealfall Konsequenzen dieser Entwicklung sein (Göckel et al. 2003: 58). ${ }^{19}$

Das Verhalten der Führungskräfte - insbesondere derjenigen auf der untersten Ebene - wird zum kritischen Erfolgsfaktor für leistungsorientierte Vergütungssysteme. In vielen Unternehmen ist zu beobachten, dass Führungskräfte Zielvereinbarungs- oder Beurteilungssysteme unterlaufen, weil die einzelne Führungskraft den Nutzen dieser Instrumente im Vergleich insbesondere zum zeitlichen Aufwand als zu gering ansieht. Führungskräfte müssen also zunächst einmal vom Nutzen des Systems überzeugt sein - oder überzeugt werden. Darüber hinaus müssen Führungskräfte aber auch für die neuen Aufgaben qualifiziert werden (Göckel et al. 2003: 56 f.). Wie anspruchsvoll die Aufgabe für Führungskräfte ist, gemeinsam mit den Beschäftigten eine für beide Parteien als "fair« und "gerecht" akzeptierbare Regelung in Vergütungsfragen zu finden, zeigt anschaulich das Beispiel der Einführung des »Neuen Bewertungs- und Bezahlungssystems (NBBS) bei der Telekom im Jahre 200I. Rund 25.000 und damit ca. 25 Prozent der Beschäftigten nahmen das vereinbarte Beanstandungsrecht in Anspruch und ließen ihre Eingruppierung von bundesweit zwölf paritätischen Kommissionen überprüfen. ${ }^{20}$ Das oben skizzierte Führungsmodell einer intensivierten, dezentralen und konsensorientierten Bearbeitung der Interessengegensätze zwischen dem staatlichen Arbeitgeber und den Beschäftigten durch die unteren Führungskräfte ist der eigentliche Kern der Reformbestrebungen.

\section{Betriebswirtschaftliche und-soziologische Implikationen des TVöD}

Die neuen Herausforderungen, die auf den öffentlichen Dienst zukommen, sind damit ungefähr erkennbar:

I9 Die Autorin enthält sich einer Ausarbeitung der negativen Konsequenzen dieser Entwicklung. Es geht hier lediglich darum zu zeigen, wie die positive Wirkungskette konstruiert sein könnte, die Unternehmen und Gewerkschaften dazu bewegt, leistungsorientierte Vergütungssysteme positiv zu bewerten. Es ist offensichtlich, dass diese Entwicklung gravierende negative Konsequenzen sowohl für viele Führungskräfte wie auch für Beschäftigte haben kann.

20 Über den gesamten Prozess wurden nur wenige Informationen publiziert. Im Mai 2003 meldete ver.di (2003: 3), dass immer noch Fälle ungeklärt seien. Dass das Verhalten der Führungskräfte letztlich entscheidend für den Erfolg der Neuregelungen des TVöD sein wird, scheint auch die Auffassung der dbb tarifunion zu sein. Im Mittelpunkt des Symposiums "Mitarbeiterführung im öffentlichen Dienst « am 31.05.2005 in Köln stand die Umsetzung des TVöD. Der Erste Vorsitzende Stöhr stellt in seinem Einführungsvortrag fest: »Der einzelne Arbeitnehmer wird [durch den TVöD, CR] stärker als früher in seiner persönlichen Leistung und Leistungsbereitschaft wahrgenommen und bewertet. [...] Dieses Wahrnehmen und Bewerten gehört ganz sicher zum Kern sachgerechter Führung im öffentlichen Dienst.« (Stöhr 2005: 3). Offenbar wollten die Gewerkschaften auch einen entsprechenden Anspruch auf Qualifizierung für Führungskräfte im TVöD verankern, konnten sich mit dieser Idee aber nicht durchsetzen. 
I. Personalrät|inn|e|n und Arbeitgeber|innen müssen zunächst die Rahmenbedingungen des TVöD für die spezifischen, in ihrem Betrieb vorkommenden Aufgaben konkretisieren. Die bisherigen Erfahrungen in vergleichbaren Situationen beispielsweise bei der Telekom oder der Deutschen Bahn zeigen, dass üblicherweise beide Seiten extrem vorsichtig agieren. Die Beteiligung von Beratungsunternehmen und Anwält|inn|en oftmals in doppelter Besetzung auf Arbeitgeber|innenseite und auf der Seite des Personalrats - ist in solchen Organisationen üblich. Der Informationsbedarf ist immens, der Aushandlungsprozess zieht sich häufig über ein Jahr und mehr. Entsprechend entstehen oft umfangreiche und komplexe Betriebsvereinbarungen. Der Zeitrahmen für die Umsetzung der leistungsorientierten Vergütung im Rahmen des TVöD bis 2007 ist insofern durchaus angemessen. Von großer Bedeutung wird sein, ob die Verhandlungspartner|innen sich auf möglichst einfache Lösungen einigen können. Regelungen, die für Führungskräfte und Mitarbeiter|innen nur schwierig nachvollziehbar sind, führen zu massiven Akzeptanzproblemen.

2. Das Ausmaß der Herausforderung, Führungskräfte vom Nutzen einer leistungsorientierten Vergütung zu überzeugen und entsprechend zu schulen, lässt sich heute noch kaum erahnen. Schätzungsweise betreffen die notwendigen Vorbereitungen ungefähr 500.000 Führungskräfte. ${ }^{2 \mathrm{I}}$ Die Akzeptanz betriebswirtschaftlicher Methoden und neuer Management-Instrumente wird in der öffentlichen Verwaltung nicht höher sein als in privatwirtschaftlichen Unternehmen. ${ }^{22}$ Mit einem im Vergleich zu privatwirtschaftlichen Unternehmen eher niedrigen Aufwand von ca. 2.00o€ für Information und Schulung pro Führungskraft gerechnet, ergeben sich erhebliche Kosten.

3. Die Beschäftigten müssen informiert und idealerweise auch an der Ausarbeitung spezifischer Lösungen beteiligt werden. Führungskräfte müssen zu ihren Mitarbeiter|inne|n ein in vielen Fällen sicher neues Verhältnis des Vertrauens und der Offenheit aufbauen. Dabei gelten hinsichtlich der Akzeptanz des Systems ähnliche Restriktionen wie für die Führungskräfte. ${ }^{23}$

4. Schließlich müssen die richtigen Ziele, Kenn- und Messgrößen entwickelt werden, die den Leistungsbegriff für die Organisationen und Unternehmen des öffentlichen Sektors operationalisieren. ${ }^{24}$ Die Verankerung eines strategischen Managements, die

2I Im öffentlichen Dienst arbeiten ca. zweieinhalb Millionen Beschäftigte, weitere drei Millionen arbeiten in Organisationen und Unternehmen, die derzeit in Anlehnung an den BAT bezahlen. Die Schätzung beruht also auf einer Führungsspanne von zehn Mitarbeiter|inne|n.

22 Empirisch - zum Beispiel im Dialog mit den Beschäftigten - findet man vor allem zwei Argumente, die die Akzeptanzprobleme verstärken: I. Im öffentlichen Dienst sei Leistung nicht messbar; 2. Der öffentliche Dienst habe keine eindeutigen Kund|inn|en, daher sei der Inhalt von »Leistung" nicht zu definieren. Battis (2003: 33) sieht ein besonderes Akzeptanzproblem bei den Beamt|inn|en, einer Beschäftigtengruppe, die bereits heute durch eine besonders hohe Quote an Klagen gegen ihren "Dienstherrn« auffällt.

$23 \mathrm{Zu}$ spezifischen Erfahrungen im öffentlichen Bereich vgl. Tondorf et al. (2004: I83 ff.).

24 Tondorf et al. (2004) zeigen, dass und unter welche Rahmenbedingungen Zielvereinbarungen einen Beitrag zur Reform der öffentlichen Verwaltung leisten können. 


\begin{abstract}
Ableitung von Output-Zielen, ${ }^{25}$ die die Beschäftigten realisieren können und wollen, und die Messung der Zielerreichung, die einen Lernprozess der Organisation ermöglicht, wären zentrale Schritte für eine Reform des öffentlichen Dienstes. Die angestoßenen Verbesserungsprozesse müssten dann systematisch gesteuert und verstetigt werden. Um einen kontinuierlichen Wandel zu ermöglichen, müssten dann die Strukturen des öffentlichen Dienstes erheblich flexibilisiert werden.
\end{abstract}

Gerade für die Umsetzung eines zielorientierten Managements und eines organisationalen Lernprozesses ist allerdings die leistungsorientierte Vergütung eher eine Hypothek: Die Vergütungsrelevanz senkt die Akzeptanz von Zielvereinbarungen erheblich, weil Führungskräfte und Mitarbeiter|innen nun nicht nur ein Steuerungsproblem - die Formulierung »richtiger« Ziele -, sondern auch ein monetäres Verhandlungsproblem lösen müssen. ${ }^{26}$

Es ist also völlig verfehlt, die Veröffentlichung des skizzierten »Sondierungsergebnisses« oder den Abschluss des TVöD als »Reform« zu bezeichnen. Allein die neuen tariflichen Rahmenbedingungen werden den öffentlichen Dienst nicht verändern. Der Versuch, im Kontext einer angespannten Haushaltslage eine Reform sozusagen zum Nulltarif über einige tarifvertragliche Änderungen durchzuführen, wird scheitern. Die Einführung einer leistungsorientierten Vergütung in der öffentlichen Verwaltung macht selbst Veränderungen notwendig und könnte einen Reformprozess möglicherweise unterstützen - unter Umständen wird sie jedoch Reformprozesse auch gefährden. Denn die für eine erfolgreiche Einführung leistungsorientierter Vergütung notwendigen Investitionen sind für die öffentliche Verwaltung nicht budgetiert. In Anbetracht der finanziellen Situation der öffentlichen Haushalte erscheint es unwahrscheinlich, dass diese überhaupt finanzierbar sind. ${ }^{27}$ Eine gescheiterte Einführung wird spürbar negative Auswirkungen auf die Reformbereitschaft im öffentlichen Dienst haben und möglicherweise eine Stagnation des Modernisierungsprozesses nach sich ziehen.

25 Oft führt die Einführung von Zielvereinbarungen in Unternehmen zu einer stärkeren OutputOrientierung, da Ziele in der Regel eher den Output als den Input messen. Dass allerdings diese Chance den Verfasser|inne|n des TVöD bewusst war, wird schon durch die in diesem Zusammenhang völlig widersinnige Vereinbarung zur Verlängerung der Arbeitszeit in Frage gestellt. Offensichtlich handelt es sich bei der Arbeitszeit um eine reine Input-Größe.

26 Die Wahrscheinlichkeit, dass - spieltheoretisch gesprochen - die Verhandlungspartner|innen in einem Zielvereinbarungsgespräch ein Gefangenendilemma mit der Möglichkeit einer kooperativen Win-Win-Lösung sehen und diese realisieren, ist viel höher, wenn die Vereinbarung der Ziele keine monetären Konsequenzen hat. Im Fall von Zielvereinbarungsgesprächen mit Verbindung zur Vergütung haben Mitarbeiter|innen hingegen einen deutlichen Vorteil, wenn sie Informationsasymmetrien nutzen, um die Wahrscheinlichkeit einer Prämie zu erhöhen. Tondorf et al. (2004: 190) konstatieren: „Die Risiken des Scheiterns oder der Fehlsteuerung sind bei diesem Zielvereinbarungstyp [vergütungsrelevante Zielvereinbarung, CR] vergleichsweise hoch."

27 Vgl. auch Battis (2003: 32 f.). 


\section{Die "Reform « als strategischer Fehler}

Auch bei genauer Analyse zeigt sich, dass der Tarifabschluss für den öffentlichen Dienst aus Sicht der Beschäftigten rein defensiven Charakter hat. An keinem Punkt wurden Verbesserungen der Arbeitsbedingungen durchgesetzt. Die gefundene Lösung schwächt das System der Flächentarifverträge sowohl hinsichtlich der Arbeitszeitregelung als auch in Bezug auf die Ausgestaltung der variablen Vergütung. Offen ist, wie viele Gewerkschaftsmitglieder nicht akzeptieren werden, dass ihre Gewerkschaften nur noch innerhalb der eigenen Klientel umverteilen.

Auf betrieblicher Ebene entstehen neue Konfliktlinien, die die Betriebs- und Personalrät|inn|e|n schwächen werden. Die Notwendigkeit zum Abschluss von Dienst- bzw. Betriebsvereinbarungen wertet Personal- bzw. Betriebsrät|inn|e|n zwar auf. Erste Lösungen auf betrieblicher Ebene werden jedoch zögerliche Betriebs- und Personalrät|inn|e|n schnell unter Handlungsdruck setzen. Im gedeckelten System des TVöD findet ein Nullsummenspiel statt: Nur wenn bestimmte Beschäftigte weniger bekommen, können andere mehr bekommen. Viele Beschäftigte - statistisch um die 50 Prozent - werden also zu faktischen Verlierer|inne|n. Auch wenn es zunächst nur ein Prozent Lohneinbuße sein wird, ist zu erwarten, dass sich die Einführung leistungsbezogener Entgeltsysteme auf die Akzeptanz von Personalund Betriebsrät|inn|e|n durch die Beschäftigten negativ auswirken wird.

Aus heutiger Sicht lässt sich die Einigung auf die Inhalte des TVöD nur als taktischer Sieg um den Preis eines strategischen Fehlers verstehen. Mit der Inszenierung als Reformkraft gelang es ver.di, Zustimmung auch aus dem konservativen und neoliberalen Lager zu erzielen. Der Verweis auf den TVöD mag noch einige Monate für die Widerlegung von Blockadevorwürfen taugen. Langfristig wird sich zeigen, dass die "Reform« eines gesamtwirtschaftlichen Sektors keine Gewerkschaftsaufgabe ist. Ver.di und die weniger bekannte dbb tarifunion haben die Verantwortung für das Gelingen eines Projektes übernommen, ohne Einfluss auf die zentralen Steuerungsgrößen Budget und Zeit der Führungskräfte und Mitarbeiter|innen für Information und Schulung zu haben. Dass man diesen Preis zu zahlen bereit war, weist einerseits auf strategische Defizite hin. Hier zeigt sich aber auch, welchen Handlungsdruck der Reformdiskurs in Deutschland derzeit für die Akteur|inn|e|n und Institutionen des traditionellen Sozialstaats erzeugt.

\section{Literatur}

Akerlof, George A. / Yellen, Janet L. (Hg.) (1986): Efficiency Wage Models of the Labor Market, Cambridge: Cambridge University Press

Battis, Ulrich (2003): Wundertüte oder Mogelpackung? - Eine kritische Betrachtung der Reformvorschläge, in: dbb akademie, Richtige Weichenstellung oder falsches Signal. Der öffentliche Dienst der Zukunft - Vorschläge der Regierungskommission NRW zur Reform des öffentlichen Dienstes in der Diskussion, Dokumentation einer Fachtagung am 27. März 2003 in Köln, S. 29-37

Coleman, James S. (1995): Grundlagen der Sozialtheorie. Band I: Handlungen und Handlungssysteme, München/Wien 
Dressler, Matthias (2000): Variable Anreizsysteme motivieren, in: Personalwirtschaft, H. 9, S. $40-46$

Göckel, Wilfried/Kratz, Felix/Wilken, Bernhard (2003): Einführung flexibler Gehälter ohne Mehrkosten, in: Personalwirtschaft, H. II, S. 54-58

Kaar, Robert van het/Grünell, Marianne (200I): Variable Pay in Europe, in: EIROnline, Id: TNoIO420IS, http://www.eiro.eurofound.eu.int/200I/o4/study/tnoIO420Is.html (Letzter Zugriff: 02.08.2005)

Kurdelbusch, Antje (2002): Variable Vergütung in deutschen Großunternehmen - Entgeltsysteme zwischen Flexibilisierung und Flächentarifvertrag, Bochum (Online-Dissertation), http://deposit.ddb.de/cgi-bin/dokserv?idn=969849834 (Letzter Zugriff: 02.08.2005)

Neuberger, Oswald (1989): Assessment Center - Ein Handel mit Illusionen?, in: Lattmann, Charles (Hg.), Das Assessment-Center-Verfahren der Eignungsbeurteilung. Sein Aufbau, seine Anwendung und sein Aussagegehalt, Heidelberg, S. 29I-307

Schulten, Thorsten (1998): Debis AG Agreement a First in Industry-related Services, in: EIROnline, Id: DE9803257F, http://www.eiro.eurofound.eu.int/1998/03/feature/ de9803257f.html (Letzter Zugriff: 02.08.2005)

Sprenger, Reinhard K. (2005): Vertrauen führt. Worauf es in Unternehmen wirklich ankommt, Frankfurt am Main

Stöhr, Frank (2005): Mitarbeiterführung im öffentlichen Dienst. Einführungsvortrag gehalten auf dem Symposium »Mitarbeiterführung im öffentlichen Dienst« der dbb tarifunion am 31.05.2005 in Köln

Tondorf, Karin (1997): Leistungszulagen als Reforminstrument. Neue Lohnpolitik zwischen Sparzwang und Modernisierung, Berlin

Tondorf, Karin/Bahnmüller, Reinhard/Klages, Helmut (2004): Steuerung durch Zielvereinbarungen. Anwendungspraxis, Probleme, Gestaltungsüberlegungen, Berlin

ver.di (2003): Deutsche Telekom AG: Handfeste Erfolge mit NBBS. Mehr als 20.00o Beanstandungen erfolgreich bearbeitet, in: komm. Telekommunikation - Informationstechnologie, Jg. 2, H. 5, S. 3

Williamson, Oliver E. (1990): Die ökonomischen Institutionen des Kapitalismus. Unternehmen, Märkte, Kooperationen, Tübingen

Zander, Ernst/Femppel, Kurt (2000): Leistungsorientierte Vergütung, Köln 